PHYSICAL REVIEW C 73, 059901(E) (2006)

\title{
Erratum: Scaling functions and superscaling in medium and heavy nuclei [Phys. Rev. C 73, 047302 (2006)]
}

\author{
A. N. Antonov, M. V. Ivanov, M. K. Gaidarov, E. Moya de Guerra, P. Sarriguren, and J. M. Udias \\ (Received 19 April 2006; published 16 May 2006) \\ DOI: 10.1103/PhysRevC.73.059901 \\ PACS number(s): 25.30.Fj, 21.10.Ft, 21.60.-n, 99.10.Cd
}

Equation (6) of the above article contains a typographical error. In the last term, the argument of the nucleon momentum distribution $n(k)$ is more appropriate to be $k$ than $p$. The correct form of Eq. (6), which describes the weight function $\left|G\left(\bar{k}_{F}\right)\right|^{2}$ is given by

$$
\left|G\left(\bar{k}_{F}\right)\right|^{2}=-\left.\frac{1}{n_{0}\left(\bar{k}_{F}\right)} \frac{d n(k)}{d k}\right|_{k=\bar{k}_{F}} .
$$

A similar form of $\left|G_{p(n)}\left(\bar{k}_{F}\right)\right|^{2}$ already exists in Eq. (21). The results and conclusions of the article are not affected. 